\title{
The forgotten merits of GIC restorations: a systematic review
}

\author{
Hawshan Abdulrahman Mustafa ${ }^{1}$ (D) Ana Prates Soares ${ }^{1} \cdot$ Sebastian Paris $^{1} \cdot$ Karim Elhennawy $^{2} \cdot$ Paul Zaslansky $^{1}$
}

Received: 30 January 2020 / Accepted: 8 May 2020 / Published online: 8 June 2020

(C) The Author(s) 2020

\begin{abstract}
Objective To reevaluate proven strengths and weakness of glass ionomer cements (GICs) and to identify agreement versus conflicting evidence in previous reports regarding the transition between GIC and the tooth, and the existence of an "interphase". Materials and methods Relevant electronic databases (PubMed, Embase via Ovid and Medline via Web of science) were searched for publications of evidence relating to the transition zone at the GIC-tooth interphase. Studies were examined and grouped according to characteristics of GIC-tooth attachment area quantified by X-ray and optical microscopy techniques in 2D and 3D.

Results Inclusion criteria comprised of in vitro studies that showed images of the conventional GIC-tooth substrate attachments using at least one of the following techniques: SEM, CLSM, or $\mu \mathrm{CT}$. The search identified 419 studies, from which 33 were included. Ten studies demonstrated the existence of an interphase layer and five studies quantified the layer thickness $(1-15 \mu)$. Twenty-nine publications studied different failure modes of the GIC-tooth interphase. Eleven studies described discontinuities inside the GIC bulk.

Conclusion The GIC-tooth interphase attributes evolve with time. Good attachment is evident even under compromised surface preparation. The GIC-tooth attachment area is resistant to acidic dissolution as compared to both tooth and GIC bulk. In general, studies revealed mostly intact GIC-tooth interphases with only some cracked interphases.

Clinical significance GIC bonds to the tooth structure and forms an acid resistant attachment zone that might enhance caries inhibition. Due to fluoride release and ease of use, GIC provides a cost effective treatment, ideal for low income or high caries populations.
\end{abstract}

Keywords Glass ionomer cement $\cdot$ Interface $\cdot$ Interphase layer $\cdot$ GIC-tooth attachment $\cdot$ Integrity and interaction interphase

Paul Zaslansky

paul.zaslansky@charite.de

Hawshan Abdulrahman Mustafa

hawshan.mustafa@charite.de

Ana Prates Soares

ana.prates-soares@charite.de

Sebastian Paris

sebastian.paris@charite.de

Karim Elhennawy

karim.elhennawy@charite.de

1 Department of Operative and Preventive Dentistry, Charité-Universitätsmedizin Berlin, Aßmannshauser Str. 4-6, 14197 Berlin, Germany

2 Department of Orthodontics, Dentofacial Orthopedics and Pedodontics, Charité-Universitätsmedizin Berlin, Germany, Aßmannshauser Str. 4-6, 14197 Berlin, Germany

\section{Introduction}

Glass ionomer cement (GIC) is an acid-base biomaterial consisting of an acid-degradable fluoro-aluminosilicate glass powder, a polymeric acid dissolved in water and tartaric acid [1]. It has been in extensive clinical use since 1972, when the first commercial cements were developed [2]. GIC forms strong chemical bonds to the dental hard tissues (dentine and enamel) as well as metals in clinical use (e.g., orthodontic brackets) [3]. It is able to adhere to prepared tooth structure in the moist environment of the mouth and has been used to seal marginal gaps at the interfaces between restoration and the tooth substrate $[4,5]$. Due to a reactive chemistry, GIC releases fluoride continuously with a desirable positive effect on tooth tissues while possibly inhibiting caries formation [6]. GIC reportedly leads to fewer allergic reactions, and reduced dental sensitivity with low cytotoxicity and mutagenicity than resin-modified glass ionomer cements, ceramics, gold alloys, and composite materials as alternatives to amalgam [7]. 
However, due to reduced mechanical and esthetic properties, the use of GIC is limited [8].

GIC has been used successfully in various forms. As a liner, it can be used to replace calcium hydroxide and other similar base materials in all cavities under class I and II composite restorations [9]. It is considered as an alternative sealing material replacing mineral trioxide aggregate (MTA) due to less crown discoloration [10]. GIC was used as periorestoration in root caries and when reinforced with hydroxyapatite, it enhances fibroblast proliferation and their attachment [11]. Additionally, it buffers bacterial acidic byproducts and increases the $\mathrm{pH}$ of the medium and it totally inhibits the growth of $S$. mutans and S. sanguinis [12]. Endodontically, GIC was used for cementation of glass fiber posts and showed similar push out bond strength tests as compared with resin-modified glass ionomer cements and self-adhesive resin cement with a similar depth of dentine penetration [13, 14]. When used to bond orthodontic brackets to tooth surfaces, it leads to significantly less white spot formation on enamel as compared with di-acrylate orthodontic cements [15]. An important application is the use as a fissure sealant in situations where drying of the tooth and moisture control are a problem. This is particularly useful for patients with high-risk caries [7, 16, 17]. Consensus on caries management has led to recommend that GIC be used in atraumatic restorative treatment as a restoration in primary and permanent teeth $[18,19]$. In a 1-year randomized controlled trial, atraumatic restorative treatment was compared to standard dental care and was found to be more cost effective [20]. Indeed, GIC-based dental materials are associated with lower secondary caries [21]. GIC was even advocated as a bulk material in the cavity, due to minimum shrinkage and wet surface attachment [22]. However, GIC lacks color stability and is not as strong as metallic restorations and evidence shows that GIC is brittle and easily cracks, due to the low flexural strength [23]. Thus, there is a lack of clarity about the state of interaction between GIC and tooth substrate and when it fails at this interface.

Recent evidence has shown that in spite of compromised properties, clinical meta-analysis works favor GIC-based materials for cervical restorations, where they show excellent often superior longevity as compared with other treatment alternatives $[24,25]$. The main advantage of this material over the alternatives lies in the interaction with the tooth substrate. GIC attaches to the underlying dentine or enamel by one or several mechanisms. These include (1) GIC-tooth interlocking - it has been proposed that GIC eliminates the smear layer and partially erodes the substrate surfaces providing mechanical retention; (2) adsorption - this mechanism assumes that chemical bonds (ionic, hydrogen) or forces (Van der Waals) act together; and (3) diffusionvarious authors propose that mobile ions exchange at the GIC-tooth transition zone where an "interphase" of gradual transition between the two material phases is formed [26]. With time, after GIC application, chemical bonds are formed between carboxyl groups (COO-) in the GIC and the tooth substrate hydroxyapatite mineral particles comprising calcium and phosphate [22]. Any etched detached ions (calcium and phosphate) from the tooth are trapped in the unreacted GIC cement and form a distinct zone/layer at GIC-tooth interphase. This interphase layer contains calcium, phosphate, aluminum, fluoride, and silica depending on the composition and on GIC interaction with the substrates [27]. The interphase interaction layer between GIC and tooth substrates has been given various, often-confusing terms [18]. The GIC sealing and its interphase with tooth substrates have been characterized using polarized-light microscopy [28, 29], scanning electron microscopy (SEM) [30, 31], transmission electron microscopy (TEM) [32,33], confocal laser scanning microscopy (CLSM) [34, 35], micro-computed tomography $(\mu \mathrm{CT})[36,37], \mathrm{X}$-ray photoelectron spectroscopy (XPS) $[29,30,38,39]$, energy-dispersive $X$-ray spectrometry (EDX) [40, 41], Fourier transform infrared spectroscopy (FTIR) [39, 42], Raman spectroscopy [43-45], and electron probe microanalysis [46, 47].

The present study aims to systematically review the evidence regarding the interaction interphase layer and attachment integrity between GIC and tooth substrates. Evidence for the presence of pores and cracks studied using SEM, CLSM, and $\mu \mathrm{CT}$ is surveyed to (1) better understand the morphology of GIC-tooth interaction interphase and (2) to outline proven strengths and weakness of GIC in clinical practice.

\section{Methods}

\section{Inclusion criteria}

This systematic review was limited to studies

- Showing images of conventional GIC and the attachment zone to human tooth tissue.

- In vitro studies that used at least one of the following techniques: SEM, CLSM, or $\mu \mathrm{CT}$.

\section{Exclusion criteria}

- Clinical, in situ, in vivo and animal studies.

- Use of bovine teeth.

- Studies that examined the attachment of GIC to composite or resin modified GIC.

- Studies lacking visual representation/figures showing the attachments observed by the authors. 


\section{Outcomes}

The continuity of the interphase between conventional GIC and human teeth tissue was assessed. Not all data in each study was depicted in the images published and available for inspection. Both qualitative (e.g., failure types) and quantitative (e.g., interphase interaction layer thickness) observations were assessed.

\section{Information sources}

PubMed, Embase via Ovid, and Medline via Web of Science were searched. Furthermore, reference lists of identified full texts were screened and cross-referenced. The search period was from 1 January 1978 to 29 October 2019. Neither authors nor journals were blinded to the evaluators. No language restriction was set; native speakers translated one study published in language other than English (Chinese).

\section{Search strategy}

The following search, combining four search blocks and employing Boolean operators, was adapted for each database:

( (( ( ( ( ( ((Glass ionomer cement) OR GIC) OR Conventional GIC) OR Conventional glass ionomer cement) OR restorative glass ionomer cement) OR glass ionomer filling) OR glass ionomer restoration) OR Resin modified glass ionomer cement $)))$ AND $((((($ dentin $)$ OR dentine $)$ OR Enamel) OR Tooth substrate) OR Dental hard tissue))) AND (()(()(()((Interface) OR Intermediate layer) OR (Junction between GIC and Tooth)) OR GIC Tooth binding) OR GIC tooth attachment) OR Chemical bonding) OR GIC tooth interaction zone) OR GIC marginal adaptation) OR Absorption layer))) AND $(((()((()(()((($ SEM) OR Scanning electron microscopy) OR EDX) OR Energy dispersive x-ray spectroscopy) OR CLSM) OR Confocal laser scanning microscopy) OR XPS) OR X-ray photon spectroscopy) OR FTIR) OR Fourier transform infrared spectroscopy) OR Raman spectroscopy) OR Micro-ct) OR Micro computed tomography) OR X-ray microtomography)).

\section{Data management}

For data extraction, a spreadsheet was used to collect the data across all studies.

\section{Selection process}

Titles and abstracts were screened by two authors (HM, AS), who compared their findings. In case of disagreement, titles were included to obtain full texts. Full texts were assessed independently after removal of duplicates. In cases of disagreement, studies were included after screening by content (figure and method).

\section{Data collection and analysis}

Data was collected by two evaluators (HM, AS) and doublechecked by two other evaluators (KE, PZ). Disagreements were resolved through discussion.

The following items were summarized: author details, year of publication, techniques used, storage medium, pretreatment usage, presence/analysis of enamel interphase, presence/ analysis of dentine interphase, interphase interaction layer, structural integrity, failure types observed and presence of pores in the GIC bulk.

Results were compared between studies and common and conflicting findings of different evaluators were identified. Risk of bias assessment was not performed, as the included papers spanned 40 years, during which image quality and instruments resolution changed substantially.

\section{Results}

\section{Selection criteria}

From 419 identified studies, 84 were scrutinized, and 33 studies were finally included (Fig. 1). In these, 26 different GIC materials were reported. To simplify and standardize comparability between the studies, characteristics were grouped into attributes of the interaction interphase layer, as well as measures of structural integrity (porosity, gaps at the GIC-tooth interphase, and cracking of the bulk). Figure 2 gives a graphical illustration of the main groups of features found in literature. A summary of the main observations is given in Table 1 .

\section{GIC-tooth interaction interphase}

Following placement of GIC on the tooth, during setting, a distinct interphase appears. It comprises an intermediate layer between the GIC and the tooth substrate. In 10 studies that showed this interphase layer, the time for the formation was 1 to 10 days of contact between GIC and tooth substrates [44, $45,55,62,67,68,71,72,75,76]$. It develops due to chemical interaction between the filling and tooth. This interphase comprises a layer of hybrid composition, and typically appears less dense and more transparent than both the adjacent tooth substrate and GIC bulk.

Different authors use conflicting terms to identify this interphase, with names including "interfacial layer," "distinct zone of interaction," "demineralized dentine," "acid-base resistant layer," "mineral infiltration zone," "absorption layer," "hybrid layer," "interdiffusion zone," and "intermediate layer" $[45,48,55,57,61,62,67,68,70-72,75,76]$. The interaction 


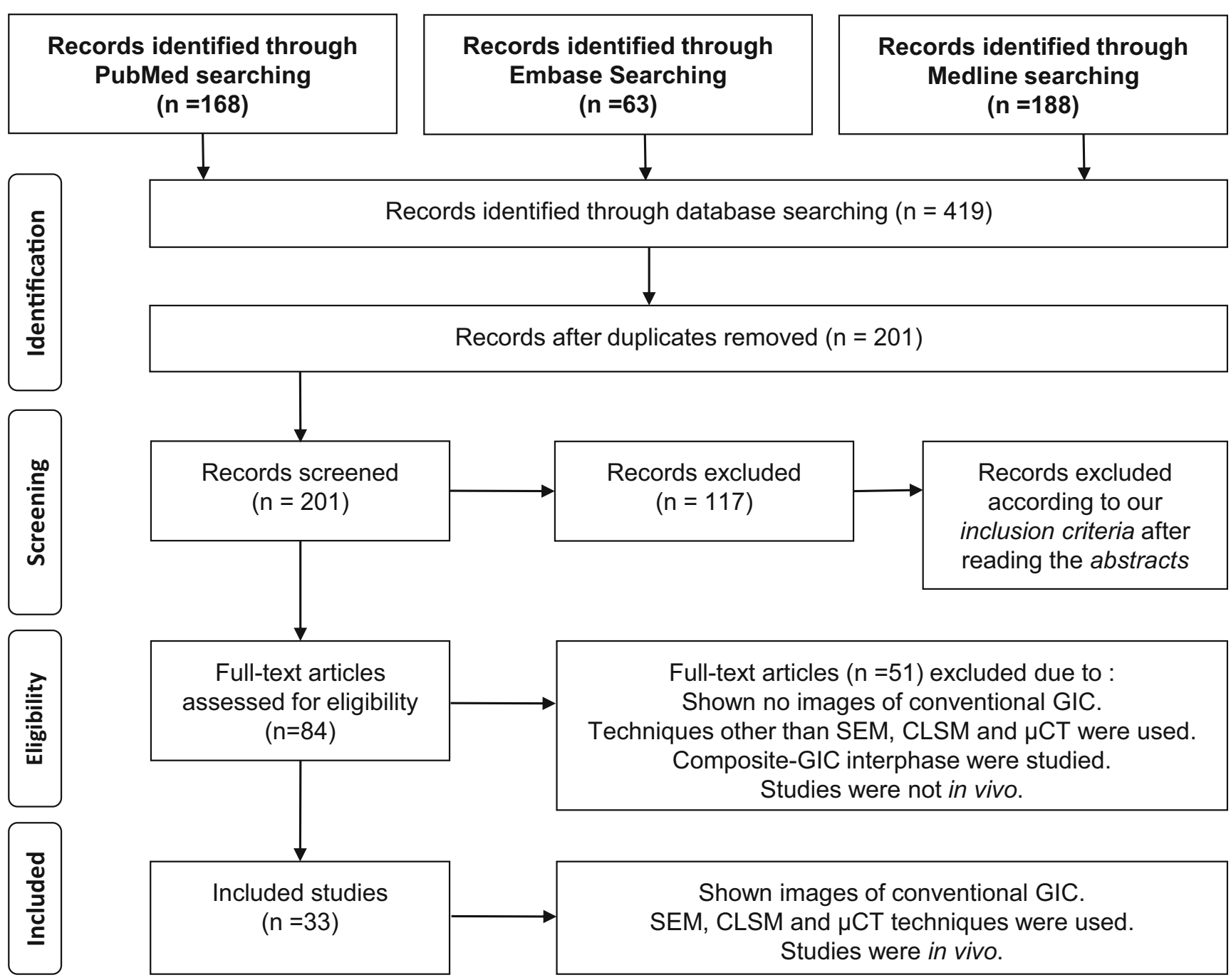

Fig. 1 Flowchart of the study. $n$ number of studies, SEM scanning electron microscopy, CLSM confocal laser scanning microscopy, $\mu$ CT microcomputed tomography

interphase forms as a result of ion exchange following dentine/enamel etching due to chemical interactions with the polyacrylic acid [8].

The extent of the interaction interphase has been the subject of several studies that reported thicknesses of $14.5 \mu \mathrm{m}$ [44], 5-7 $\mu \mathrm{m}$ [55], 2.8-3.4 $\mu \mathrm{m}$ [70], $1 \mu \mathrm{m}$ [61], 1-2 $\mu \mathrm{m}$ [62], and 0.4-0.5 $\mu \mathrm{m}$ [67]. SEM and CLSM were both used to characterize the interphase size, as reported in 11 of the studies [44, $45,55,62,67,68,70-72,75,76]$. Interestingly, 10 of the included studies (Table 1) suggested a complete lack of any interaction interphase [48-51, 56, 58, 61, 63, 74, 78].

Enamel and dentine can each form interaction interphases with GIC, only 2 studies observed interphase formation where GIC is in contact with both tooth tissues $[62,75]$. Ngo et al. [62], using the SEM, found interphases including GIC-enamel and GIC-dentine attachments. Interphases were observed either with or without acid etching (precondition) of the tooth samples. The work by Yilmaz [75] only detected a layer near the preconditioned dentine surface and found no interphase layer when GIC was applied to pristine dentine or enamel samples.

\section{Structural integrity}

From the 33 included studies, 29 examined the structural integrity of GIC restorations, including analysis of both the filling itself and the interphase with teeth substrates [44, 45, 49-51, 53-62, 64-75, 77, 78].

\section{Structural integrity of GIC-tooth interphase}

The quality of GIC attachment to the tooth is determined by the interphase integrity. The ideal restoration should exhibit intact cohesion. Interphase integrity was found to be compromised in 14 of the 29 studies. The authors reported either adhesive failure (Fig. 2d) exhibiting a complete detachment of GIC from the tooth or a mixed mode of failure (Fig. 2c), where GIC remnants remain partially attached to the tooth 


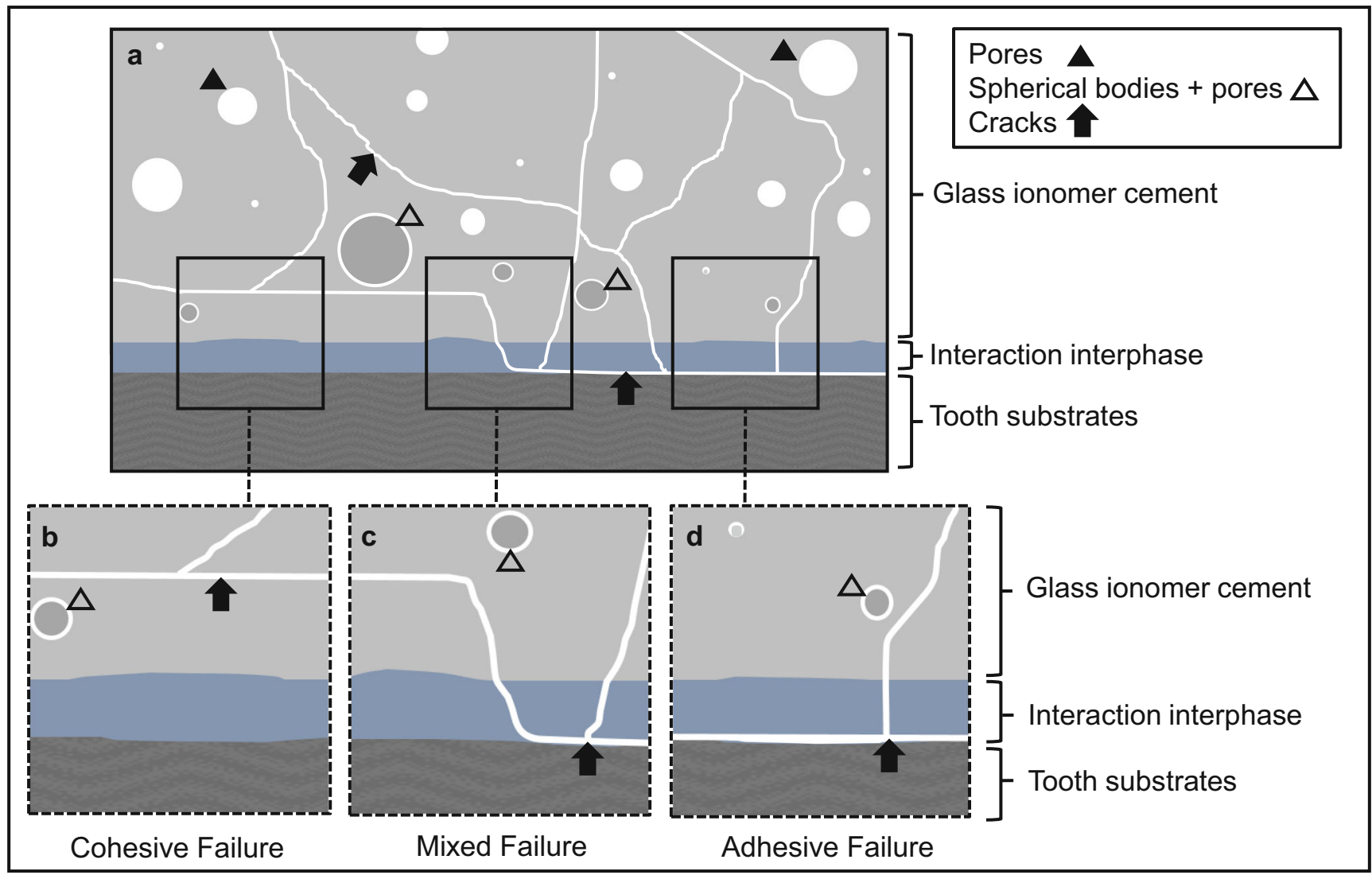

Fig. 2 A schematic illustration of the GIC characteristics analyzed in this review

substrate $[50,51,53,56,58-60,64,65,71,74,75,77,78]$. Six of the studies found complete detachment following mechanical testing $[50,51,53,65,71,77]$. Even without mechanical loading, GIC attachment may be compromised as shown by Grossman and Mickenautsch [59]. The authors found that $16 \%$ of the interphase length was detached.

GICs are well known for their self-adherence to tooth substrates and require no conditioning [44]. However, the quality of attachment of GIC to tooth substrates might change according to the use of pretreatments (e.g., etchant or conditioner). Conflicting reports exist regarding the effects that pretreatment of the tooth substrates has on interfacial integrity. Among the 33 included studies, a majority (25) used pretreatments [45, 48-53, 55-58, 60-63, 65, 68-73, 75-77]. Five studies used none $[44,54,67,74,78]$ whereas 3 studies did not mention anything about the application of pretreatments $[59,64,66]$. An intact attachment was found in 12 of the 25 studies that included pretreatment of their samples $[45,49,55$, 57, 61, 62, 68-70, 72, 73, 75]. However, intact attachments were also seen in three $[44,54,67]$ of the 5 studies where samples were not pretreated $[44,54,67,74,78]$ as shown in Table 1. This suggests that $50 \%$ of interphases of all tested GICs result in detachment, regardless of any applied pretreatment. The attachment quality was also affected by the type of tooth conditioner. Citric acid application was more favorable than EDTA [65].
A curious observation for GIC-dentine samples was that the interphase zone was more resistant to recurring acid etching. Indeed following such etching of visibly intact interphases [55, 62], the neighboring dentine and GIC were always eroded as compared to the attachment zone.

\section{Structural integrity of the bulk}

When GIC cracks are seen near the interphase with tooth structure, the fillings are classified as exhibiting cohesive failure (Fig. 2b). A lack of structural integrity in the bulk of restorations was reported in 15 studies [44, 45, 49, 54, 55, 57, 61, 62, 66-70, 72, 73]. Seven studies considered crack formation, in which 5 studies identified dehydration [49, 53, $58,73,74]$, and 2 studies associated stress during mechanical tests as main causes for structural cracking. Those authors demonstrated that cracks appear at a distance from the interphase with the tooth [50, 69]. Birkenfeld and Schulman [49] speculated that faulty sample handling and preparation might also lead to cracking. The reported cracking on GIC surfaces usually did not lead to full fracture of the GIC restoration at the base of cavity, but this is not the same as when mechanical tests were used, where force was applied until complete failure of GIC bulk occured [53,69].

In addition to cracks, the bulk of GIC contains pores. Porosity, defined as the presence of empty spaces inside 


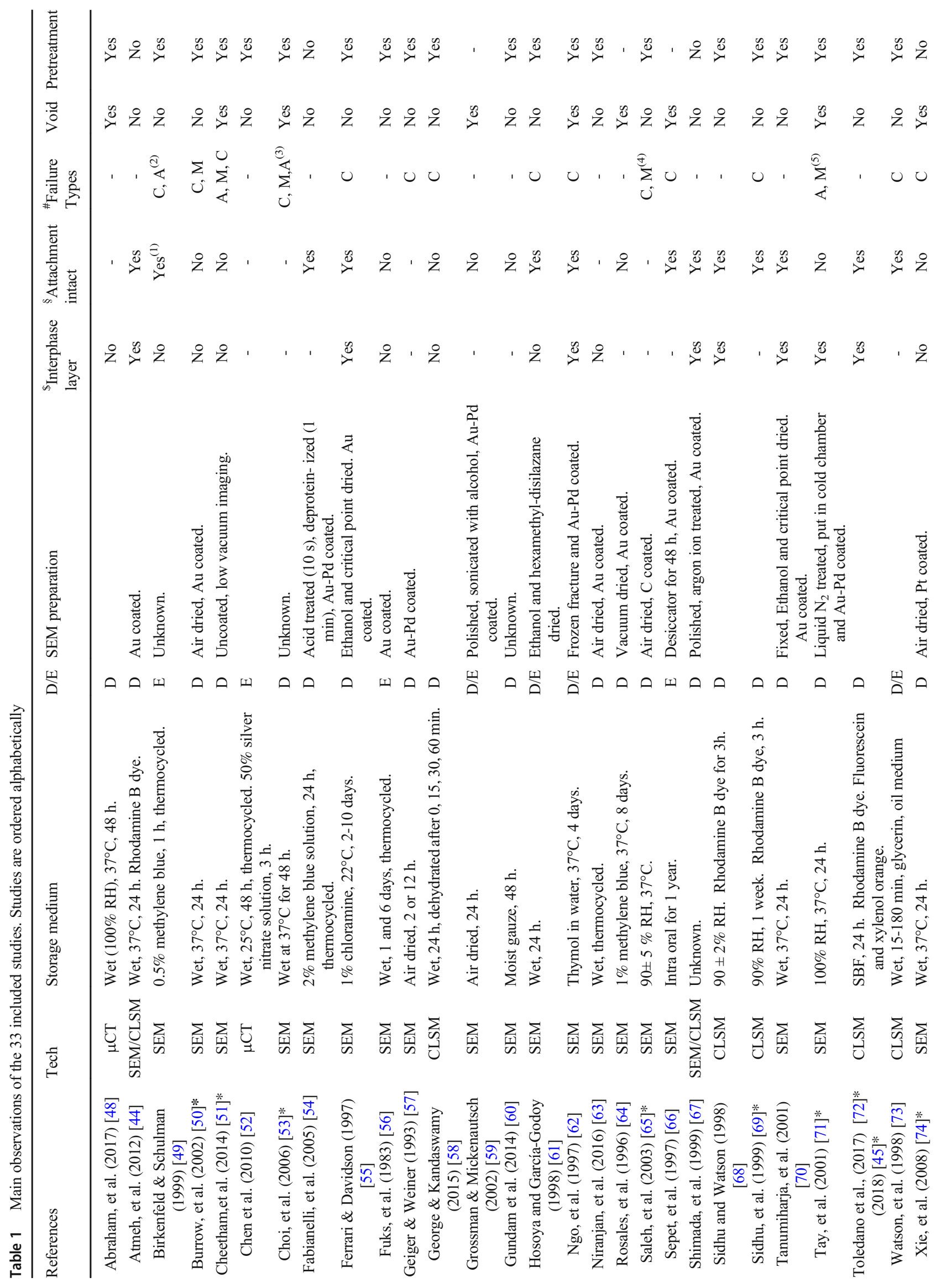




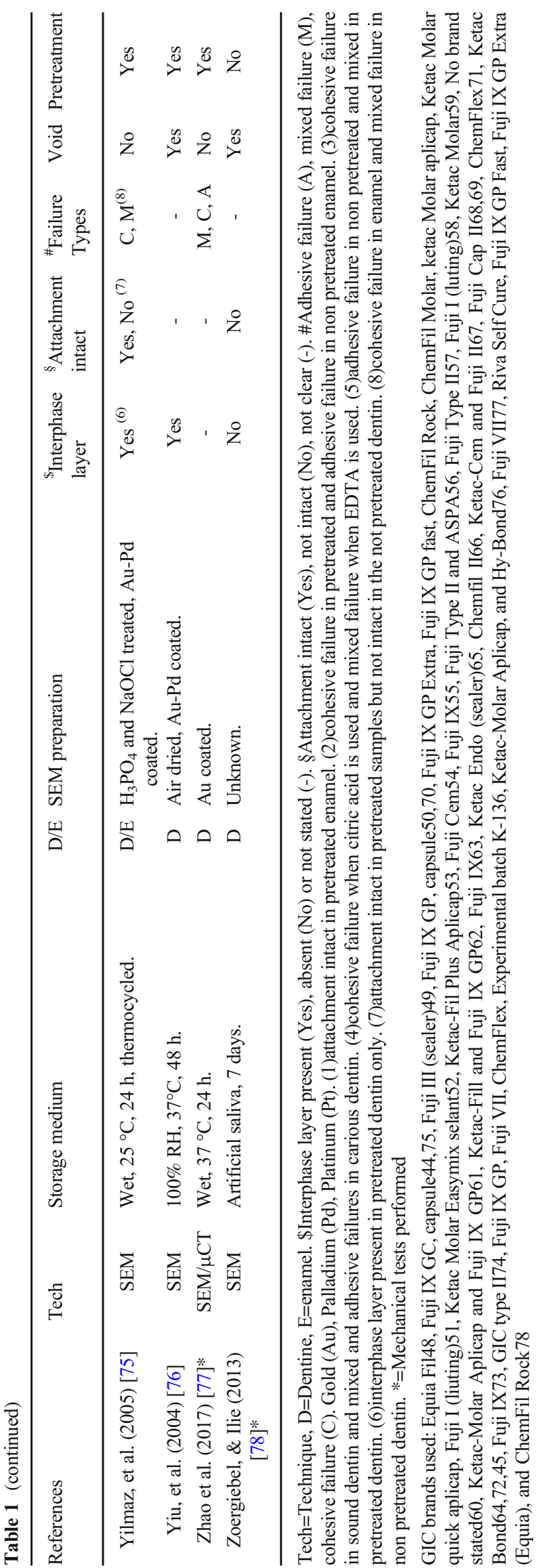

the bulk of GIC, was reported in 11 studies [48, 51, 53, 59, $62,64,66,71,74,76,78]$, but it was sometimes identified as "voids," "air bubbles," or "air inclusions." Three out of the 11 studies measured the porosity in the bulk of the restorations in either 3D [48] or 2D [59, 62]. A $\mu$ CT-based study by Abraham et al. [48] used silver nitrate infiltration to quantify the $3 \mathrm{D}$ percentage of porosity in entire fillings reporting a $0.88 \%$ prevalence. The two remaining studies used SEM to examine cut surfaces [59, 62]. Grossman and Mickenautsch [59] measured diameters of single pores $(<50 \mu \mathrm{m})$ and observed that each $500 \mu \mathrm{m}^{2}$ of GIC contains 100 to 200 pores. The pores were spread inside the bulk of GIC restoration, mostly in the lower two thirds of the restorations from middle to the bottom of the cavity [62]. All studies examined pores in dried GIC-tooth samples and found pores free from material. One study by Yiu et al. [76] reported the presence of spherical bodies inside pores in moist samples. Those spherical bodies were localized within pores close to the attachment between GIC and moist dentine. They were not seen in GIC pores several (3+) mm away from the attachment between GIC and moist dentine (Fig. 2a). These spherical bodies were found to be rich in the element silicon $(\mathrm{Si})$, which comes from $\mathrm{SiO}_{2}$, one of the main glass forming components used in the cement, deposited during secondary setting reactions of the GIC. This deposition forms a purely silica phase that has three times higher Si concentrations than the GIC matrix. The authors assume the spherical bodies are glass ionomer reaction remnants that form while in contact with water diffused from dentine. Continuation of such reactions helps GIC maturation that eventually increases the compressive strength of GIC [76].

\section{Sample preparation and imaging}

A total of 25 studies used SEM with variable storage conditions and time. The majority (21 out of 25) reported sample preparation before SEM evaluation, whereas sample preparation for SEM was not described in the remaining four. The process of dehydrating samples as a standard protocol before SEM evaluation was reported in 10 studies $[50,55,61$, 63-66, 70, 74, 76]. Different methods were used for dehydration; air drying was used in six of the studies [50, 59, 63, $64,74,76]$. The rest used either chemical and/or mechanical protocols such as serial alcohol dehydration or embedding of the samples, with or without using a critical point dryer. Five studies did not dry their samples: two of those due to the use of replicas $[49,56]$ and three others maintained the samples either frozen [62, 71] or moist [76] during scanning. Electron imaging by SEM revealed the interphase layer in seven of the 15 studies that evaluated the GIC-tooth substrate interaction region (Table 1).

CLSM was used to image samples in 8 of the reviewed studies $[44,45,58,67-69,72,73]$. Five of them used 
fluorescence staining with rhodamine B [44, 45, 68, 69, 72], and two of them used time lapse imaging to observe cement luting, dehydration, and rehydration [58, 73]. Two reports from the same research group looked at the effects of mechanical loading on the GIC-dentine attachment [45, 72]. Sidhu et al. [69] used real-time imaging of a shear test to examine GIC failure modes, which was $100 \%$ cohesive. The CLSM technique was capable of showing the interphase layer in 5 of the 8 studies (Table 1).

Imaging with $\mu \mathrm{CT}$ was reported in 3 studies; two of them evaluated whole samples [52,67], whereas the third assessed sliced samples [77]. Pixel sizes varied between 9 and $14 \mu \mathrm{m}$ and virtual slices of the samples were presented in the publications. The $\mu \mathrm{CT}$ technique demonstrated pores but was not capable of showing the interphase layer.

\section{Discussion}

Despite the drawbacks, GICs contribute significantly to the range of solutions available to the treating dental surgeon. Many clinicians advocate limited if any use for these materials, for diverse reasons not all of which are substantiated with scientific findings. Understanding the details of when GIC advantages outweigh known shortcomings is thus of merit and may have important financial consequences. It is also in line with recent clinical studies that show excellent performance of cervical lesions treated with GIC-based materials $[24,25]$. All studies surveyed here observed that GIC has a reproducible ability to attach to both enamel and dentine surfaces. Despite a well-documented use and antibacterial efficacy, GIC is heavily criticized because of its low stressbearing ability, color instability, and solubility. However, GIC also has a long track record with some excellent results reported for specific dental conditions.

Thirty-three studies were finally included as a basis for this systematic review. All included works reported on detailed structural features of the GIC-tooth interphase and restoration integrity. Importantly, an array of methods and consequent different sample preparation conditions lead to heterogeneity in the findings, often resulting in conflicting experimental observations. Here, we attempt to standardize and reconcile reports from a wide range of research groups.

\section{Interphase attributes}

\section{Agreement in the literature}

An interaction interphase layer is formed and seen when GIC comes into contact with pretreated dentine $[45,55,67,68,71$, $75,76]$. It has been proposed that such pretreatment exposes collagen and facilitates diffusion of GIC into the porous demineralized substrate [72]. That may be a reason why many studies pretreated dentine, although GIC is self-etching and may not require pretreatment to form a sound interface with the tooth substrates.

Following GIC application, the interphase layer appears after $1-10$ days $[44,45,55,62,67,68,71,72,75,76]$. Two studies claim that the interphase layer contains elements originating from both GIC and the tooth substrates, basing this conclusion on evidence as presented by transmission electron microscopy and Raman spectroscopy [44, 71]. The interphase interaction layer was shown to comprise mainly fluoridatedcarbonate-apatite, with the tooth serving as source of apatite that leaches out from dentine/enamel across the GIC-tooth attachment region [44, 57, 67]. Specifically, Geiger and Weiner [57] postulated that the interphase layer either (1) forms by dissolution and precipitation of fluoride salts of the GIC carbonate apatite crystals together with the tooth substrate, etched by the polyacrylic acid or (2) forms by diffusion of fluoride ions from GIC into the crystals of dental substrates. However, it is not clear if this takes place on the tooth side or the GIC side of the tooth-filling interface.

The interphase is permeable to fluorescent dyes and water such that flow from wet dentine to the ion-rich GIC matrix region is straightforward $[68,72,73,76]$. This presumably contributes to the establishment of the interphase layer.

\section{Disagreement in the literature}

Only one study reported formation of an interaction interphase layer in GIC placed on enamel. Ngo et al. [62] were the only authors among the 9 studies examining GIC-enamel interfaces that showed a distinct interaction layer. Although all other authors examined the interface with high-resolution SEM, none found this layer. We cannot rule out that a reason for this discrepancy might be sample preparation differences: Ngo et al. [62] used cryo-freezing of the sample and a low temperature SEM, revealing a very thin interphase layer, appearing somewhat like an imaging artifact. Those same authors also observed that the interaction layer was absent in cases when GIC was poorly attached to the tooth substrates and it did not exist adjacent to voids. Further high-resolution work is probably needed to confirm or refute the existence of an interaction interphase near enamel. It seems likely that similar to dentine, when enamel apatite is exposed to etching and comes into close contact with freshly mixed GIC, an interphase will form.

There also remains uncertainty regarding the precise location of the interaction interphase layer related to dentine. While some authors believe that it resides within the smear layer, others believe it may be located on/within the surface of the demineralized dentine [71]. The location appears to vary by the protocol of etching used, e.g., with or without substrate pretreatment. Thus, further work is needed to determine the 
relationship between substrate pretreatment and GIC, as well as the exact location and composition of the interphase layer.

\section{Continuity of attachment at the interface}

\section{Agreement in the literature}

Many authors reported excellent adaptation and a tight contact of GIC with the tooth substrates, suggesting good wetting during placement. Such fillings typically form a continuous intact contact with the tooth tissues $[44,45,54,55,61,62$, 66-70, 72, 73]. The intimate contact between material and substrate seems to improve with pretreatment of dentine and enamel surfaces prior to the application $[61,62,65,66,73$, 75]. Several authors reported that applying pressure to the GIC-dentine zone improved the sealing ability and reduced the interphase porosity $[45,72]$. The $\mathrm{pH}$ of the etchant and the duration of application of the pretreatments affected the GIC-tooth attachment. Thus, the attachment between the enamel and GIC was intact when the enamel surface is pretreated $[61,62,73,75]$, but appeared flawed when enamel was not pretreated $[49,75]$.

The GIC-dentine interaction attachment is acid resistant as shown by Ferrari and Davidson [55] and Ngo et al. [62]. Dentine with an interphase zone was less affected by acid etching and was less susceptible to acidic material removal as compared to the GIC and tooth substrates surrounding the attachment area [55, 62]. This resistance to etching may be due to incorporation of polyacrylic acid that may be less soluble at low $\mathrm{pH}$. This suggests that GICs may induce chemical 'tempering' and may help improve resistance to future caries attacks, at least on the short term. There have been speculations that the GIC-tooth substrate attachment might act as a barrier against lactic acid dissolution of tooth tissues. Some authors even report bactericidal affects that reduce accumulation of microorganisms and may help hinder secondary caries $[57,64]$.

\section{Disagreement in the literature}

While many authors agreed that adaptation between GIC and tooth substrates in pretreated samples is improved, some authors reported disrupted attachment observations, despite pretreatment $[56,58,60,71]$. This appears to be due to induced mechanical stress on these samples, since the authors report thermocycling [56], changes in humidity [58,60] or mechanical testing designed to characterize failure patterns [71]. While mechanical tests lead to partial (mixed failure) or complete (adhesive failure) separation of GIC from tooth substrates, there appear to be significant effects of sample preparation: non-intact interfaces were always reported in studies where SEM was used [50, 51, 53, 65, 71, 74, 77, 78], whereas CLSM studies, which do not require dehydration for imaging, showed intact attachment [45, 72]. One group reported that GIC performance is actually better than the contact formed by resin-modified GIC due to the absence of the HEMA component. Those authors hypothesized that resin-reinforced GIC were inferior since the HEMA absorbs water, leading to separation from the substrate [68]. Sample preparation for imaging may thus have a strong effect on previous reports in the literature therefore new evidence is called for.

\section{GIC structural failure and defects}

\section{Agreement in the literature}

GIC is a hydrophilic material and stays intact in a $80 \%$ relative humidity environment; any increase or decrease in humidity leads to a change in the GIC structure [74]. It swells and disintegrates when fully saturated with water, and it shrinks and cracks when dehydrated [73]. Studies that used CLSM or SEM without sample dehydration prior to imaging did not report any cracks $[56,62,67,71,76]$. However, applying forces during mechanical tests produces stress inside the bulk of the material that leads to the breakage of bonds between the matrix and GIC particles [50, 69]. It has been shown that dehydration of dentine produces high stress [79, 80], which may contribute to cracking at the interface. Many factors thus affect the bonding durability of the GIC-tooth interphase and forces developing in either the GIC or the tooth substrates influence crack formation [50, 53, 73]. A large number of studies employed sample dehydration when observing crack formation over time $[58,73]$ or as a part of sample dehydration for SEM imaging purposes [49, 53, 74]. Cracks are mostly cohesive, appearing at the bulk of GIC close to the attachment with tooth substrates due to the low cohesive strength of GIC [55, 57, 58, 61, 62, 66, 69, 73, 74]. Indeed, cohesive failure was predominant among the included studies in the present review reported three times as much as adhesive failure, regardless of pretreatment, or of whether mechanical testing was applied, or if samples were stored wet or dry (Table 1). Cohesive failure inside the bulk of GIC suggests that (1) GIC-tooth flexural strength is higher than in the bulk of GIC and (2) although GIC cracks in the bulk, it remains attached to the tooth substrates where a thin layer of GIC material covers the tooth substrate surfaces $[55,62,66,69,71]$.

Another recurring structural defect was the rather frequent presence of pores inside the bulk of GIC. Material handling procedures and consequent air entrapment were found to be correlated to the appearance of pores or voids in GIC [48, 51, $59,62,66,74]$. The prevalence of pores is higher when GIC is mixed using automated machines rather than by manual mixing [62]. Pores either at the interphase or in the bulk of GIC are likely to increase the chance of fracture leading to restoration failure [50, 51, 59, 64, 74]. 


\section{Disagreement in the literature}

There are reports of cracks related to GIC that include mixed and adhesive failures, the latter being less common $[49-51,53,65,71,75,77]$. It is possible that the difference between failure modes relates to the specifics of the GIC brands used; this is because cement manufacturers use different glass particle types, sizes, geometries, and overall different glass filler content. Cracks will typically initiate at the interfaces between glass particles and the matrix which is the weaker link as compared to the interface of the GIC and the substrate [62]. GIC cracks cannot be repaired; however, adding water to freshly cracked GIC might reduce the gap size even if does not completely heal it $[68,73]$. One group [53] used considerations of the Griffith-Irwin theory to argue that cohesive cracks observed near the interface with tooth tissue may actually represent weak interfacial bonding. However, some of the bond strengths reported were very high (almost $40 \mathrm{MPa}$ ) as compared to composite bonding systems suggesting excellent attachment of GIC to the tooth substrate. Of additional concern are the presence of pores and the occasional observation of material inside pores. The latter is rarely mentioned although many authors reported the presence of GIC pores. Only one report showed that spherical bodies do occupy GIC pores near the GICdentine attachment area, as observed when the tooth is humid [76]. Pores may certainly contribute to the emergence of cracks in the bulk and hence further work is necessary to explore the possible contribution of porosity to the preferential cohesive failure of GIC.

\section{Study limitation}

The present study surveyed research spanning multiple decades during which electron and confocal imaging microscopy was completely revolutionized. It is thus likely that technological limitations of either GIC production or the imaging methods used may have strongly influenced the reported results. One of the limitations in our study was the partially descriptive nature of many observations, often limited to the outer surface of the samples investigated. Much of the data reported is qualitative and many images are of compromised quality. Adding complementary techniques including TEM, XRD, FTIR, and Raman imaging may therefore help resolve some of the conflicting reports in the literature. We note that the studies included multiple generations and different GIC types $(n=26)$ where composition varies. Nevertheless, comparisons of the different studies included make it possible to revisit some of the prevailing conceptions regarding this group of materials.

\section{Reappraisal of images}

All papers were re-appraised to allow comparison of the reported information, but only a small subset of images was available for direct comparison. It was visibly clear that the quality of micrographs and images increased in the more recent works. This is due to the technological developments and increasing utilization of automated computerized systems with improved and more rapid detection systems used to examine GIC-tooth substrate interphases. However, $36 \%$ of the included studies (both old and new studies) lacked significant amounts of data to be able to properly assess and compare the analyses provided. Specifically, some results were not well documented in the pictures provided; some of the captions did not clearly describe the presented photos; and different papers by different authors did not report all background technical information (e.g., dehydration) that is pertinent to understanding critically important effects on the results shown.

\section{Future study recommendations}

Newer technology and high sensitivity detectors, improved computer-aided electron, and confocal imaging show great promise to better understand basic properties of the GIC-tooth interface. While $\mu \mathrm{CT}$ studies pave the way to quantify $3 \mathrm{D}$ data down to the micrometer lengthscale, the small difference in density between materials and minute dimensions of the interphase render the task difficult to image these materials using conventional $\mu \mathrm{CT}$. Phase-contrast enhanced $\mu \mathrm{CT}$ as is available from specialized instruments (synchrotron radiation facilities, e.g., ESRF, Grenoble, France, [81]) may provide additional 3D insights using tomography methods based on edge-enhanced radiography obtained e.g. from laserlike sources in large X-ray facilities. Such future measurements [81] with accentuated interphases may in turn reveal details regarding intact GIC restorations obtained in a clinically relevant setting.

SEM is a reliable source for the evaluation of GIC-tooth interphase morphology; however, it is generally destructive and requires dehydration that is likely to change the real structural relations of the sample. Therefore, using complementary techniques where samples can be examined wet, with no further dehydration steps, is recommended. Other options include FIB-SEM and cryo-based system where the hydration state of the sample may be preserved. Most studies in the literature tested only limited time-spans such that stability and dynamic changes are poorly investigated. New studies mapping material attributes over time, ideally for more than 3 months are needed. 


\section{Conclusion}

Despite many advantages, GIC has compromised mechanical properties, specifically low strength. Yet, it appears to present good bonding to the tooth structure, and due to fluoride release and ease of use, it provides a cost effective treatment, mainly for cervical or smallsized (incipient) tooth cavities [25]. The main findings of the present review include:

- Formation of GIC-tooth Interaction interphase layer may take 1-10 days as a result of a chemical ion diffusion process, in which ions from GIC and from the tooth substrate are exchanged.

- The thickness of the interaction interphase layer ranges from 1 to $15 \mu$. The thickness and location of this layer varies with the mode of application, duration, and types of pretreatment.

- The GIC-tooth attachment area is notably resistant to acidic dissolution.

- Although GIC is self-adhesive to tooth substrates, pretreatments tends to improve the quality of attachment, possibly by improving wetting.

- GIC is susceptible to cohesive cracking in the bulk, often not far from the interface with the tooth tissue.

- The GIC-dentine interphase changes over time due to interactions between the restorative material, tooth and water as shown by die penetration.

Funding information Open Access funding provided by Projekt DEAL. The work of Hawshan Abdulrahman Mustafa was supported by the German Academic Exchange Service (DAAD). The work of Ana Prates Soares was supported by the Elsa Neumann Stipendium des Landes Berlin.

\section{Compliance with ethical standards}

Conflict of interest The authors declare that they have no conflict of interest.

Ethical approval This article does not contain any studies with human participants or animals performed by any of the authors.

Informed consent For this type of study, formal consent is not required.

Open Access This article is licensed under a Creative Commons Attribution 4.0 International License, which permits use, sharing, adaptation, distribution and reproduction in any medium or format, as long as you give appropriate credit to the original author(s) and the source, provide a link to the Creative Commons licence, and indicate if changes were made. The images or other third party material in this article are included in the article's Creative Commons licence, unless indicated otherwise in a credit line to the material. If material is not included in the article's Creative Commons licence and your intended use is not permitted by statutory regulation or exceeds the permitted use, you will need to obtain permission directly from the copyright holder. To view a copy of this licence, visit http://creativecommons.org/licenses/by/4.0/.

\section{References}

1. Wilson AD (1991) Glass-ionomer cement-origins, development and future. Clin Mater 7:275-282. https://doi.org/10.1016/02676605(91)90070-v

2. Smith DC (1998) Development of glass-ionomer cement systems. Biomaterials 19:467-478. https://doi.org/10.1016/s0142-9612(97) 00126-9

3. Wilson AD, Kent BE (1971) The glass-ionomer cement, a new translucent dental filling material. J Appl Chem Biotechnol 21: 313. https://doi.org/10.1002/jctb.5020211101

4. Albers HF (2002) Tooth-colored restoratives: principles and techniques. BC Derek, Hamilton ISBN: 1-55009-155-7

5. Yan Z, Sidhu SK, Carrick TE, McCabe JF (2007) Response to thermal stimuli of glass ionomer cements. Dent Mater 23:597600. https://doi.org/10.1016/j.dental.2006.05.001

6. Sakaguchi RL, Powers JM (2012) Craig's restorative dental materials. Elsevier/Mosby, St. Louis ISBN:978-0-3230-8108-5

7. European C, Directorate General for H, Consumers (2015) The safety of dental amalgam and alternative dental restoration materials for patients and users. https://doi.org/10.2772/42641

8. Mount GJ (2002) An atlas of glass-ionomer cements: a clinician's guide. Martin Dunitz, London ISBN 0-203-21545-1

9. Wilson NH, Mjor IA (2000) The teaching of class I and class II direct composite restorations in European dental schools. J Dent 28: 15-21. https://doi.org/10.1016/s0300-5712(99)00055-x

10. Peng CF, Yang Y, Zhao YM, Liu H, Xu Z, Zhao DH, Qin M (2017) Long-term treatment outcomes in immature permanent teeth by revascularisation using MTA and GIC as canal-sealing materials: a retrospective study. Int J Paediatr Dent 27:454-462. https://doi. org/10.1111/ipd.12282

11. Thomas B, Gupta K (2017) In vitro biocompatibility of hydroxyapatite-added GIC: an SEM study using human periodontal ligament fibroblasts. J Esthet Restor Dent 29:435-441. https:// doi.org/10.1111/jerd.12317

12. Nedeljkovic I, De Munck J, Slomka V, Van Meerbeek B, Teughels W, Van Landuyt KL (2016) Lack of buffering by composites promotes shift to more cariogenic bacteria. J Dent Res 95:875-881. https://doi.org/10.1177/0022034516647677

13. Pereira JR, da Rosa RA, So MVR, Afonso D, Kuga MC, Honorio HM, do Valle AL, Vidotti HA (2014) Push-out bond strength of fiber posts to root dentin using glass ionomer and resin modified glass ionomer cements. J Appl Oral Sci 22:390-396. https://doi.org/ 10.1590/1678-775720130466

14. Lorenzetti CC, Bortolatto JF, Ramos ATPR, Shinohara AL, Saad JRC, Kuga MC (2019) The effectiveness of glass ionomer cement as a fiber post cementation system in endodontically treated teeth. Microsc Res Tech 82:1191-1197. https://doi.org/10.1002/jemt. 23268

15. Marcusson A, Norevall LI, Persson M (1997) White spot reduction when using glass ionomer cement for bonding in orthodontics: a longitudinal and comparative study. Eur J Orthod 19:233-242. https://doi.org/10.1093/ejo/19.3.233

16. Beauchamp J, Caufield PW, Crall JJ, Donly K, Feigal R, Gooch B, Ismail A, Kohn W, Siegal M, Simonsen R, Frantsve-Hawley J (2008) Evidence-based clinical recommendations for the use of pit-and-fissure sealants - a report of the American Dental Association Council on Scientific Affairs. J Am Dent Assoc 139: 257-268. https://doi.org/10.14219/jada.archive.2008.0155

17. American Academy of Pediatric Dentistry. Clinical Affairs Committee - Restorative Dentistry S (2012) Guideline on pediatric restorative dentistry. Pediatr Dent 34:173-180

18. Nicholson JW (2016) Adhesion of glass-ionomer cements to teeth: a review. Int J Adhes Adhes 69:33-38. https://doi.org/10.1016/j. ijadhadh.2016.03.012 
19. Banerjee A, Frencken JE, Schwendicke F, Innes NPT (2017) Contemporary operative caries management: consensus recommendations on minimally invasive caries removal. Br Dent J 223: 215-222. https://doi.org/10.1038/sj.bdj.2017.672

20. Tonmukayakul U, Arrow P (2017) Cost-effectiveness analysis of the atraumatic restorative treatment-based approach to managing early childhood caries. Community Dent Oral Epidemiol 45:92100. https://doi.org/10.1111/cdoe.12265

21. Sidhu SK (2016) Glass-ionomers in dentistry. Springer International Publishing, Cham. https://doi.org/10.1007/978-3319-22626-2

22. Aboush YE, Torabzadeh H (2000) Clinical performance of Class II restorations in which resin composite is laminated over resinmodified glass-ionomer. Oper Dent 25:367-373

23. Wilson AD, Nicholson JW (2005) Acid-Base cements : their biomedical and industrial applications. ISBN: 0-521-37222-4r

24. Peumans M, De Munck J, Mine A, Van Meerbeek B (2014) Clinical effectiveness of contemporary adhesives for the restoration of non-carious cervical lesions. A systematic review. Dent Mater 30:1089-1103. https://doi.org/10.1016/j.dental.2014.07. 007

25. Schwendicke F, Gostemeyer G, Blunck U, Paris S, Hsu LY, Tu YK (2016) Directly placed restorative materials: review and network meta-analysis. J Dent Res 95:613-622. https://doi.org/10.1177/ 0022034516631285

26. Davidson CL, Mjör IA (1999) Advances in glass-ionomer cements. Quintessence Pub. Co., Chicago ISBN: 0-86715-360-1

27. Sennou HE, Lebugle AA, Gregoire GL (1999) X-ray photoelectron spectroscopy study of the dentin-glass ionomer cement interface. Dent Mater 15:229-237. https://doi.org/10.1016/s0109-5641(99) 00036-6

28. Trairatvorakul C, Kladkaew S, Songsiripradabboon S (2008) Active management of incipient caries and choice of materials. J Dent Res 87:228-232. https://doi.org/10.1177/ 154405910808700301

29. Wu YH, Hutton JE, Marshall GW (1997) In vitro enamel demineralization and the marginal gap of simulated cast restorations with three different cements. J Prosthodont 6:96-103. https://doi.org/10. 1111/j.1532-849x.1997.tb00074.x

30. Lin A, McIntyre NS, Davidson RD (1992) Studies on the adhesion of glass-ionomer cements to dentin. J Dent Res 71:1836-1841. https://doi.org/10.1177/00220345920710111401

31. Abdalla AI (2000) Morphological interface between hybrid ionomers and dentin with and without smear-layer removal. J Oral Rehabil 27:808-814. https://doi.org/10.1046/j.1365-2842. 2000.00601.x

32. Yip HK, Tay FR, Ngo HC, Smales RJ, Pashley DH (2001) Bonding of contemporary glass ionomer cements to dentin. Dent Mater 17: 456-470. https://doi.org/10.1016/s0109-5641(01)00007-0

33. Coutinho E, Cardoso MV, De Munck J, Neves AA, Van Landuyt KL, Poitevin A, Peumans M, Lambrechts P, Van Meerbeek B (2009) Bonding effectiveness and interfacial characterization of a nano-filled resin-modified glass-ionomer. Dent Mater 25:13471357. https://doi.org/10.1016/j.dental.2009.06.004

34. Sidhu SK, Pilecki P, Sherriff M, Watson TF (2004) Crack closure on rehydration of glass-ionomer materials. Eur J Oral Sci 112:465469. https://doi.org/10.1111/j.1600-0722.2004.00155.x

35. Sauro S, Watson T, Moscardo AP, Luzi A, Feitosa VP, Banerjee A (2018) The effect of dentine pre-treatment using bioglass and/or polyacrylic acid on the interfacial characteristics of resin-modified glass ionomer cements. J Dent 73:32-39. https://doi.org/10.1016/j. jdent.2018.03.014

36. Zakizadeh P, Marshall SJ, Hoover CI, Peters OA, Noblett WC, Gansky SA, Goodis HE (2008) A novel approach in assessment of coronal leakage of intraorifice barriers: a saliva leakage and micro-computed tomographic evaluation. J Endod 34:871-875. https://doi.org/10.1016/j.joen.2008.04.005

37. Oglakci B, Kazak M, Donmez N, Dalkilic EE, Koymen SS (2020) The use of a liner under different bulk-fill resin composites: 3D GAP formation analysis by x-ray microcomputed tomography. J Appl Oral Sci Rev FOB 28:e20190042. https://doi.org/10.1590/ 1678-7757-2019-0042

38. Coutinho E, Yoshida Y, Inoue S, Fukuda R, Snauwaert J, Nakayama Y, De Munck J, Lambrechts P, Suzuki K, Van Meerbeek B (2007) Gel phase formation at resin-modified glassionomer/tooth interfaces. Dent Res 86:656-661. https://doi.org/10. $1177 / 154405910708600714$

39. Falsafi A, Mitra SB, Oxman JD, Ton TT, Bui HT (2014) Mechanisms of setting reactions and interfacial behavior of a nano-filled resin-modified glass ionomer. Dent Mater 30:632643. https://doi.org/10.1016/j.dental.2014.02.025

40. Ryan AK, Mitchell CA, Orr JF (2002) Fracture mechanics analysis of the dentine-luting cement interface. Proc Inst Mech Eng H J Eng Med 216:271-276. https://doi.org/10.1243/09544110260138763

41. Gjorgievska E, Nicholson JW, Grcev AT (2012) Ion migration from fluoride-releasing dental restorative materials into dental hard tissues. J Mater Sci Mater Med 23:1811-1821. https://doi.org/10. 1007/s10856-012-4653-Z

42. Mitra SB, Lee CY, Bui HT, Tantbirojn D, Rusin RP (2009) Longterm adhesion and mechanism of bonding of a paste-liquid resinmodified glass-ionomer. Dent Mater 25:459-466. https://doi.org/ 10.1016/j.dental.2008.09.008

43. Papagiannoulis L, Kakaboura A, Eliades G (2002) In vivo vs in vitro anticariogenic behavior of glass-ionomer and resin composite restorative materials. Dent Mater 18:561-569. https://doi.org/10. 1016/s0109-5641(01)00090-2

44. Atmeh AR, Chong EZ, Richard G, Festy F, Watson TF (2012) Dentin-cement interfacial interaction: calcium silicates and Polyalkenoates. J Dent Res 91:454-459. https://doi.org/10.1177/ 0022034512443068

45. Toledano M, Osorio R, Osorio E, Cabello I, Toledano-Osorio M, Aguilera FS (2018) In vitro mechanical stimulation facilitates stress dissipation and sealing ability at the conventional glass ionomer cement-dentin interface. J Dent 73:61-69. https://doi.org/10.1016/ j.jdent.2018.04.006

46. Knight GM, McIntyre JM, Craig GG, Mulyani (2007) Electron probe microanalysis of ion exchange of selected elements between dentine and adhesive restorative materials. Aust Dent J 52:128-132. https://doi.org/10.1111/j.1834-7819.2007.tb00477.x

47. Knight GM, McIntyre JM, Craig GG, Mulyani, Zilm PS, Gully NJ (2007) An in vitro investigation of marginal dentine caries abutting composite resin and glass ionomer cement restorations. Aust Dent J 52:187-192. https://doi.org/10.1111/j.1834-7819.2007. tb00487.x

48. Abraham SB, Gaintantzopoulou MD, Eliades G (2017) Cavity adaptation of water-based restoratives placed as liners under a resin composite. Int J Dent 2017:1-8. https://doi.org/10.1155/2017/ 5957107

49. Birkenfeld LH, Schulman A (1999) Enhanced retention of glassionomer sealant by enamel etching: a microleakage and scanning electron microscopic study. Quintessence Int (Berlin, Germany: 1985) 30:712-718

50. Burrow MF, Nopnakeepong U, Phrukkanon S (2002) A comparison of microtensile bond strengths of several dentin bonding systems to primary and permanent dentin. Dent Mater 18:239-245. https://doi.org/10.1016/s0109-5641(01)00041-0

51. Cheetham JJ, Palamara JEA, Tyas MJ, Burrow MF (2014) Evaluation of the interfacial work of fracture of glass-ionomer cements bonded to dentin. J Mech Behav Biomed Mater 29:427-437. https://doi.org/10.1016/j.jmbbm.2013.09.020 
52. Chen X, Cuijpers V, Fan M, Frencken JE (2010) Marginal leakage of two newer glass-ionomer-based sealant materials assessed using micro-CT. J Dent 38:731-735. https://doi.org/10.1016/j.jdent.2010. 05.018

53. Choi K, Oshida Y, Platt JA, Cochran MA, Matis BA, Yi K (2006) Microtensile bond strength of glass ionomer cements to artificially created carious dentin. Oper Dent 31:590-597. https://doi.org/10. 2341/05-108

54. Fabianelli A, Goracci C, Bertelli E, Monticelli F, Grandini S, Ferrari M (2005) In vitro evaluation of wall-to-wall adaptation of a self-adhesive resin cement used for luting gold and ceramic inlays. J Adhes Dent 7:33-40

55. Ferrari M, Davidson CL (1997) Interdiffusion of a traditional glass ionomer cement into conditioned dentin. Am J Dent 10:295-297

56. Fuks AB, Hirschfeld Z, Grajower R (1983) Marginal adaptation of glass-ionomer cements. J Prosthet Dent 49:356-360. https://doi.org/ 10.1016/0022-3913(83)90277-9

57. Geiger SB, Weiner S (1993) Fluoridated carbonatoapatite in the intermediate layer between glass ionomer and dentin. Dent Mater 9:33-36. https://doi.org/10.1016/0109-5641(93)90102-v

58. George L, Kandaswamy D (2015) A confocal microscopic evaluation of the dehydration effect on conventional, resin reinforced powder/liquid and paste to paste glass ionomer luting cements. J Int Oral Health 7:28-32

59. Grossman ES, Mickenautsch S (2002) Microscope observations of ART excavated cavities and restorations. S Afr Dent J 57:359363

60. Gundam S, Patil J, Venigalla BS, Yadanaparti S, Maddu R, Gurram SR (2014) Comparison of marginal adaptation of mineral trioxide aggregate, glass ionomer cement and intermediate restorative material as root-end filling materials, using scanning electron microscope: an in vitro study. J Conserv Dent 17:566-570. https://doi.org/ 10.4103/0972-0707.144606

61. Hosoya Y, Garcia-Godoy F (1998) Bonding mechanism of KetacMolar Aplicap and Fuji IX GP to enamel and dentin. Am J Dent 11: 235-239

62. Ngo H, Mount GJ, Peters MC (1997) A study of glass-ionomer cement and its interface with enamel and dentin using a low-temperature, high-resolution scanning electron microscopic technique. Quintessence Int (Berlin, Germany : 1985) 28:63-69

63. Niranjan B, Shashikiran ND, Singla S, Thakur R, Dubey A, Maran S (2016) A comparative microleakage evaluation of three different base materials in Class I cavity in deciduous molars in sandwich technique using dye penetration and dentin surface interface by scanning electron microscope. J Indian Soc Pedod Prev Dent 34: 324-330. https://doi.org/10.4103/0970-4388.191410

64. Rosales JI, Vallecillo M, Osorio R, Bravo M, Toledano M (1996) An in vitro comparison of micro leakage in three glass ionomer cements used as retrograde filling materials. Int Dent J 46:15-21

65. Saleh IM, Ruyter IE, Haapasalo MP, Orstavik D (2003) Adhesion of endodontic sealers: scanning electron microscopy and energy dispersive spectroscopy. J Endod 29:595-601. https://doi.org/10. 1097/00004770-200309000-00013

66. Sepet E, Aytepe Z, Oray H (1997) Surface texture and enamelrestoration interface of glass ionomer restorations. J Clin Pediatr Dent 21:231-235

67. Shimada Y, Kondo Y, Inokoshi S, Tagami J, Antonucci JM (1999) Demineralizing effect of dental cements on human dentin. Quintessence Int (Berlin, Germany: 1985) 30:267-273

68. Sidhu SK, Watson TF (1998) Interfacial characteristics of resinmodified glass-ionomer materials: a study on fluid permeability using confocal fluorescence microscopy. J Dent Res 77:17491759. https://doi.org/10.1177/00220345980770091101

69. Sidhu SK, Sherriff M, Watson TF (1999) Failure of resin-modified glass-ionomers subjected to shear loading. J Dent 27:373-381. https://doi.org/10.1016/s0300-5712(98)00057-8

70. Tanumiharja M, Burrow MF, Cimmino A, Tyas MJ (2001) The evaluation of four conditioners for glass ionomer cements using field-emission scanning electron microscopy. J Dent 29:131-138. https://doi.org/10.1016/s0300-5712(00)00056-7

71. Tay FR, Smales RJ, Ngo H, Wei SH, Pashley DH (2001) Effect of different conditioning protocols on adhesion of a GIC to dentin. J Adhes Dent 3:153-167

72. Toledano M, Osorio R, Cabello I, Osorio E, Toledano-Osorio M, Aguilera FS (2017) Oral function improves interfacial integrity and sealing ability between conventional glass ionomer cements and dentin. Microsc Microanal 23:131-144. https://doi.org/10.1017/ S1431927617000010

73. Watson TF, Pagliari D, Sidhu SK, Naasan MA (1998) Confocal microscopic observation of structural changes in glass-ionomer cements and tooth interfaces. Biomaterials 19:581-588. https://doi. org/10.1016/s0142-9612(97)00140-3

74. Xie H, Zhang F, Wu Y, Chen C, Liu W (2008) Dentine bond strength and microleakage of flowable composite, compomer and glass ionomer cement. Aust Dent J 53:325-331. https://doi.org/10. 1111/j.1834-7819.2008.00074.x

75. Yilmaz Y, Gurbuz T, Kocogullari ME (2005) The influence of various conditioner agents on the interdiffusion zone and microleakage of a glass lonomer cement with a high viscosity in primary teeth. Oper Dent 30:105-112

76. Yiu CK, Tay FR, King NM, Pashley DH, Sidhu SK, Neo JC, Toledano M, Wong SL (2004) Interaction of glass-ionomer cements with moist dentin. J Dent Res 83:283-289. https://doi.org/ $10.1177 / 154405910408300403$

77. Zhao IS, Mei ML, Zhou ZL, Burrow MF, Lo EC, Chu CH (2017) Shear bond strength and remineralisation effect of a casein phosphopeptide-amorphous calcium phosphate-modified glass ionomer cement on artificial "caries-affected" dentine. Int J Mol Sci 18:1-10. https://doi.org/10.3390/ijms18081723

78. Zoergiebel J, Ilie N (2013) An in vitro study on the maturation of conventional glass ionomer cements and their interface to dentin. Acta Biomater 9:9529-9537. https://doi.org/10.1016/j.actbio.2013. 08.010

79. Forien JB, Fleck C, Cloetens P, Duda G, Fratzl P, Zolotoyabko E, Zaslansky P (2015) Compressive residual strains in mineral nanoparticles as a possible origin of enhanced crack resistance in human tooth dentin. Nano Lett 15:3729-3734. https://doi.org/10.1021/acs. nanolett. 5 b00143

80. Forien JB, Zizak I, Fleck C, Petersen A, Fratzl P, Zolotoyabko E, Zaslansky P (2016) Water-mediated collagen and mineral nanoparticle interactions guide functional deformation of human tooth dentin. Chem Mater 28:3416-3427. https://doi.org/10.1021/acs. chemmater.6b00811

81. Prates Soares A, Blunck U, Bitter K, Paris S, Rack A, Zaslansky P (2020) Hard X-ray phase-contrast-enhanced micro-CT for quantifying interfaces within brittle dense root-filling-restored human teeth. J Synchrotron Radiat 27(4). https://doi.org/10.1107/ S1600577520005603

Publisher's note Springer Nature remains neutral with regard to jurisdictional claims in published maps and institutional affiliations. 